\title{
Evolution of secure services for women in England
}

\author{
Jaydip Sarkar \& Mary di Lustro
}

\begin{abstract}
SUMMARY
Patients detained at high and medium security reveal significant gender differences in the presentation of psychopathology, mental disorder and social and offending profiles. However, secure mental health services in England, like prisons, generally fail to recognise the core importance of the differing biopsychosocial development in women and the impact of life experiences on women's subsequent biopsychosocial functioning. As a consequence, women are often inadequately provided for in services dictated by the identified needs, risks and responsiveness of men. The lack of clinically appropriate facilities for women may account for the increased frequency with which women are readmitted to medium security and for their longer admissions to both high and medium secure care. New tertiary services are developing as a result of the lessons learnt while providing gender-blind care. However, further development is required to ensure that women receive services of the same quality, range and nature of those received by men.

\section{DECLARATION OF INTEREST}

None.
\end{abstract}

The aim of this article is to highlight core differences between the genders, the key factors in terms of needs and the risks associated with various mental disorders. It will trace briefly the evolution of secure services for women and will make certain recommendations that could lead to the provision of responsive, clinically effective and financially efficient services for women.

\section{What is different about women?}

\section{Mental disorder}

Gender differences in patients' manifestation of psychopathology should be neither surprising nor controversial. The genders differ in genetics, anatomy and hormones determined by evolutionary pressures. In addition, there are differences in psychosocially determined roles - both assigned and adopted - within family and sociopolitical structures. Psychiatry reflects this. There is:

- a higher prevalence of substance use disorders in men and mood disorders in women;
- variance in the age of onset of schizophrenia, with men being younger than women;

- disparity in the range and severity of symptoms (more severe manifestation of post-traumatic stress disorder (PTSD) and bipolar disorder in women); and

- differences in course and prognosis (better prognosis of schizophrenia in women), response to treatment and key risk factors (Seeman 1995).

As regards personality disorder, women receive disproportionately more diagnoses of histrionic and dependent personality disorders. Anecdotal clinical evidence and DSM-IV-TR (American Psychiatric Association 2004) suggest that $75 \%$ of patients with personality disorders are women, but the rate of borderline personality disorder is the same in both genders overall, although with more severe manifestations in women (Lynam 2007). Men have higher rates of most other personality disorders, with disproportionately high levels of antisocial and paranoid personality disorder (Lynam 2007).

\section{Crime and violence}

There are disparities along gender lines. Women constitute just $6 \%$ of the prison population and, despite recent increases in the number of violent crimes committed by women, in 2006 less than $6 \%$ were imprisoned for such crimes, compared with nearly 95\% of men (Home Office 2007). Crime statistics in England and Wales show that men are more likely than women to be convicted of a range of violent offences: women are responsible for only $6 \%$ of murders, $1.5 \%$ of attempted murders, $16 \%$ of manslaughters, $7 \%$ of woundings and $1.3 \%$ of sexual crimes (Home Office 2006). Sexual offences constitute roughly half of all serious violent offences in any one year (Bartlett 2009).

Evolutionary salient pressures appear to drive crime in women. So on one hand, the need to provide for themselves in a poverty-stricken and violent environment (Farrington 2004) may fuel higher levels of property- and drug-related offences in women (Chesney-Lind 1997; Campbell 2001; Bartlett 2009). On the other hand, when carrying out violent crimes, women are likely to be involved in 'low risk' strategies. There are proportionately
Jaydip Sarkar is a consultant forensic psychiatrist working in the secure women's services and personality disorder services for men at Arnold Lodge in Leicester, UK. His interests include neurobiology and affective neuroscience of attachment, personality and its disorders, developmental and adult trauma and psychotherapy, mental health law and gender equality. Mary di Lustro is a consultant forensic psychiatrist in the women's service at Arnold Lodge medium secure unit. Her expertise and interests are secure services for women, establishment of the women's enhanced medium secure service pilot sites, improving gender awareness and enhancing the ability to provide relational security in the workforce and the treatment of women with personality disorder. Correspondence Dr Jaydip Sarkar, East Midlands Centre for Forensic Mental Health, Arnold Lodge, Cordelia Close, Leicester LE5 OLE, UK. E-mail: jay.sarkar@nottshc. nhs.uk 
BOX 1 Gender differences in forensic psychiatry

- Mental disorder: women tend to have higher prevalence and severity of affective and trauma-related disorders and receive more diagnoses of personality disorders

- Women commit fewer crimes of all types and tend to target those with whom they are in an attachment relationship (e.g. partners, children, psychiatric staff) in acts of interpersonal violence

- Women tend to receive more mental health rather than prison disposals when they commit a crime
- Deviant behaviour in women is often conceived of by society (e.g. the criminal justice system, the mental health system) to be based on trauma and inadequacy rather than on antisocial personality disorder

- Women are more likely to receive the Mental Health Act classification of 'psychopathic disorder' (which is not an ICD-10 category) and to be detained in higher than commensurate levels of secure care

more women represented in simple and aggravated assaults than in intentional grievous bodily harm and murder (Kruttschnitt 1993) and weapons offences (Miller 1986; Steffensmeier 1993).

Some of the so-called low-risk strategies appear to involve targeting intimate others, particularly their own children or intimate sexual partners (Weizmann-Henelius 2003; Liem 2008), or displacement of aggression onto inanimate objects through arson, property damage, etc. Women in prison have higher rates than men of substance use, PTSD, personality disorders and childhood sexual abuse. They often have basic literacy skills, are likely to have been unemployed for the 5 years before imprisonment and to have children at home (Bartlett 2009). Box 1 summarises gender differences found in forensic psychiatry.

\section{Features of secure services for women}

\section{Access to services}

A greater proportion of women in high and medium security are likely to be detained under Part II of the Mental Health Act 1983 as civil patients because most are likely to have been admitted from National Health Service (NHS) facilities rather than prison (Smith 1991; Milne 1995; Women in Secure Hospitals 1999). Women are proportionately more likely to receive a hospital disposal at court (Sahota 2009). The majority of women detained in high and medium security with a personality disorder have primary diagnoses of borderline or emotionally unstable personality disorder (Milne 1995; Coid 2000). There are no significant differences between the genders in time spent in the community or in institutions (Steels 1998).

\section{Offending history}

The majority of women in secure services were convicted of arson (Women in Secure

Hospitals 1999; Sahota 2009). Evidence suggests that they are also likely to have harmed their own children or intimate others rather than strangers (Jurik 1990; Weizmann-Henelius 2003; Liem 2008). Many women in secure services have either no index offence or, if they do, are less likely to have committed violent and sexual offences (Coid 2000; Maden 2004). Women are also comparatively less likely to have prior convictions (Steels 1998) and are less likely to reoffend after discharge (Maden 2006). However, a 2009 study by Sahota et al reveals that women committed more violence than men 2 years (38\% v. 26\%) and 5 years after discharge (49\% v. 40\%). Within this the rate of arson was much higher in women and increased with the passage of time. The reconviction rate was lower in women (42\% v. 50\%), although this is suggested to be an artefact (Sahota 2009).

\section{Reasons for admission}

Many women are admitted to both levels of security because of unmanageable self-harm, suicidal acts, aggression towards hospital staff and damage to property (Bartlett 2001). This stems from the difficulties in managing high rates of suicidal behaviour and the use of increasingly restrictive and punitive practices to manage self-harm. Such patients experience the double jeopardy of being retraumatised by their care-givers through 'anti-therapeutic, demeaning and infantilising staff attitudes' (Department of Health 1992), as well as being considered 'untreatable' owing to personality disorders.

Men are more likely than women to be detained under Part III of the 1983 Act, be subject to restriction orders with a classification of mental illness, have committed serious violent acts such as homicide, have a previous forensic history and be admitted because of mental illness or sexually risky behaviours (Women in Secure Hospitals 1999).

\section{Reasons for admission of non-forensic patients into secure forensic services}

Another set of reasons for admission into higher than commensurate levels of security may lie in the hidden risks that some of these women pose to others (and themselves). One explanation for the discordant findings in the study by Sahota et al (2009) may be because most women in that medium secure hospital were discharged to a high-security hospital. This would suggest that risk was increasing when data were collected. The trend may have been different if the women had been moving down the security ladder. Also, it is suggested that as only one in four violent acts results in conviction, there is a 'hidden' criminality in this group (Sahota 2009). However, this should 
affect both genders, not just women. This may be an artefact related to a broader societal bias of viewing deviant behaviour in women as a mental disorder (Prins 1982) rooted in trauma and inadequacy, as opposed to antisociality. This may explain the reluctance of criminal justice agencies to prosecute those detained in secure hospitals, especially if aggression is against hospital property or staff and the unwillingness of mental health professionals to view women's violence as rooted in deviance rather than trauma.

\section{Evolution of secure services for women}

The evolution of secure services for women can be considered in three broad phases:

1 services based on a gender-blind model of care;

2 gender-specific services; and

3 future needs for service development.

\section{Services based on a gender-blind model of care}

Historically, men and women had been segregated within psychiatry. However, from the 1970s, within a context of greater liberalism in Western Europe and North America (Sarkar 2011), there was a trend towards normalisation and mixed wards in all psychiatric hospitals (Warner 1998). This included medium secure units, but not necessarily high-security hospitals (Bartlett 2001). Traditional psychiatric frameworks aimed at those with major mental illnesses such as schizophrenia and bipolar disorder are inadequate to capture the psychopathology of women with personality disorders. This limits the ability of staff to make sense of themes of disempowerment and rejection that are often present in women.

Staff often tend to understand problems through the prism of psychotic and affective symptoms, labelling those behaviours that do not fall within these frameworks of understanding as 'attention-seeking,, 'manipulative' and 'problematic'. These behaviours then tend to elicit more restrictive regimes of management (Lart 1999). The resultant behavioural manifestations of self-harm, staff assaults, false allegations and pathological dependency on others reflect the maladaptive coping strategies for which the women were detained in the first place. A cycle develops where elements of gender identity (mother, partner, daughter, victim, abuser, etc.) are re-enacted with staff through pathological attachment patterns and the women are ultimately retraumatised and revictimised (Welldon 1991).

Gender-blind approaches in high security led to boundary violations and the development of inappropriate physical relationships between staff and patients, which were categorically deplored by independent inquiries (Department of Health 1992).

In 1998, results of a major national audit revealed that $94 \%$ of wards were mixed gender, with two-thirds of women having to sleep in the same area as men, women having to use the same bathrooms as men and 3\% of women sleeping in areas where the only separation from men was a curtain (Warner 1998). The latter would not be applicable to women in medium secure services. Mixed-gender wards exposed extremely vulnerable women with extensive histories of sexual abuse and victimisation to some men who had been physically and sexually violent towards women (Bland 1999), are interpersonally exploitative and who may use women for personal gratifications (financial and/or sexual) (Bartlett 2001).

These results led the NHS Executive to establish a national Women's Project Group in 1999, which culminated in the publication of Secure Futures for Women: Making a Difference (Department of Health 2000a). The report endorsed the view that women-centred services should be available both in hospital and the community. These strands of the government's intentions were woven into a national strategy for the provision of mental health services that eventually resulted in the publication of Women's Mental Health: Into the Mainstream and Mainstreaming Gender and Women's Mental Health: Implementation Guidance (Department of Health 2002, 2003). Safety, privacy and dignity for women were acknowledged and the government established specific targets. These included the removal of $95 \%$ of mixed-gender accommodation from NHS trusts by 2002 and the provision of women-only lounges and living spaces (Department of Health 2000b).

In 2000 , there were 39 medium secure units, of which almost all were mixed gender, with only 14 NHS and 79 independent-sector medium secure beds in dedicated women-only services (Bartlett 2001). By 2009, there were 27 dedicated women-only medium secure services (9 independent and 18 NHS), providing 543 beds (261 in the independent sector and 282 in NHS services) (St Andrew's Healthcare 2009). There was at least one women-only service in each health region in the country, with six in the North West and only one in the South West. Of the 27 services, 19 had a gender-specific care pathway, with either a women-only rehabilitation or pre-discharge ward, or a women-only low secure or step-down service (Parry-Crooke 2009). All health and social care partners were invited to offer discrete bespoke services for women, which reflected the essential differences in women's social and offending profiles, mental distress and complex patterns of behaviour, 
BOX 2 Key drivers for evolution of genderspecific services

- Lack of privacy, dignity and respect within a gender-blind model of care

- Physical and social abuse of women by male patients

- Boundary violations and sexual relationships with staff

- Exclusion by services, leading to detention in higher than commensurate levels of secure care with regard to risk of aggression to others

with their care and treatment needs underpinned by principles of empowerment, respect and dignity (Women In Secure Hospitals 2000).

\section{Gender-specific services}

The development of gender-specific services at lower levels of security appears to have been contingent on a drastic contraction of high secure services (for other key drivers, see Box 2). Responding to criticisms that many women were detained in conditions of security greater than commensurate levels of risk, a major review in the three national high-security units recommended that additional resources be made available for the dispersal of patients who did not require this level of security to alternative settings (Department of Health 2000c). Additional funds led to the creation of 'enhanced' medium secure services to provide 'for the small number of women, currently in high secure care, who have committed severe offences or [...] who could not be catered for within existing medium secure care, but who do not need Category "B" high secure care' (Department of Health 2003: p 41). The number of women in high security fell from 345 in 1991 to 50 in 2008, with only Rampton High Secure Hospital providing care and treatment for this group. Alongside this, three women's enhanced medium secure services (WEMSS) were established in 2007, providing 61 beds: 10 at Arnold Lodge in Leicester, 45 at The Orchard in West London and 6 at The Edenfield Centre in Prestwich.

\section{Standard v. 'enhanced' services}

So what is 'enhanced' in these services? The physical security is similar to that within 'standard' medium secure services, but WEMSS offer 'enhanced' relational and procedural security to women who cannot be managed in conditions lower than medium security. The clinical presentation of women admitted to the WEMSS differs from that of women admitted to standard medium secure care (Box 3).

It is evident that women managed in standard services have primary diagnoses of severe and enduring mental illness, such as schizophrenia, affective disorder or a combination of symptoms of the two. Any additional comorbidity with personality problems is usually secondary. As a result, most 'standard' services tend to manage their patients primarily with pharmacotherapy and with strategies that utilise intensive care management for those with acute psychotic disturbances. However, the primary diagnoses of those requiring 'enhanced' care are severe personality disorders, with Axis I disorders (American Psychiatric Association 1994) being largely comorbid as secondary disorders. Although they may receive polypharmacy (Tyrer 2004), most of them require therapeutically enhanced care that is related to their attachment needs, which is an adaptation of 'relational security' (Department of Health 2009).

\section{Importance of relational safety}

Relational security has been defined as embodying high patient-to-staff ratios, time spent in face-to-face contact between staff and patients, achieving the right balance between openness and intrusion, and working towards developing high levels of trust between the clinical team and patients (Parry-Crooke 2009). Clinicians working with women know from experience that women tend to live largely in reference to various other relationships (Box 4). This referential existence within a network of relational safety sets them apart from men, who tend to have indexical lives in societies that traditionally prioritise individual rights and responsibilities that are regulated by laws. A female patient's disturbed attachments and interpersonal functioning need to be understood in the context of the section below and the sense of loss that will result if a care team tries to alter interpersonal functioning without first seeking to establish less dysfunctional attachments. In other words, staff must first try to form a meaningful relationship with the patient before trying to change how she relates to others. It is important for

BOX 3 Characteristics of women requiring an 'enhanced' medium secure service

- Significantly higher level of manifested dependency

- Significantly higher degree of complexity of therapeutic needs

- Significantly different nature and degree of risk to themselves and/or others, particularly carers and dependent others

- Significantly disorganised patterns of attachment and affect regulation that relates to the degree of chronicity in all of the above areas 
BOX 4 Factors related to women's need for attachment relationships

- The sense of self in women occurs in reference to relationship with others

- Higher temperamental levels of negative affect and lower regulative capacities imply a need for relationships, for safety and security

- Interaction of biological demands on the body with socially determined meaningful roles lead to critical periods of high risk, e.g. early childhood, menarche, pregnancy and motherhood, and menopause

any service addressing issues of relational security to appreciate that the behaviour of patients that challenges the staff team is functional. It may provide a means of communication, may serve to regulate relationships in the only way available to the patient and may provide the patient's only meaningful method of regulating their own emotions. The Department of Health (2009) has highlighted the importance and utility of relational security in patient management.

Why do female patients require attachment as a treatment approach?

Feminist psychology Traditional theories of psychological development emphasise the primacy of separation and independence from others as indicators of health and maturity. Feminist psychology states that such theories deny the positive aspects of mutuality and sensitivity to others and the fact that the 'ability to experience, comprehend, and respond to the inner state of another person is a highly complex process relying on a high level of psychological development and ego strength' (Kaplan 1984). As a feminist psychiatrist, Miller (1986: p. 16) provides an eloquent description of the differing development of relationships between the genders:

'Male society, by depriving women of the right to its major "bounty" - that is, development according to the male model - overlooks the fact that women's development is proceeding, but on another basis. One central feature is that women stay with, build on, and develop in a context of connections with others. Indeed, women's sense of self becomes very much organised around being able to make and then maintain affiliations and relationships. Eventually for many women the threat of disruption of connections is perceived not just as a loss of a relationship, but as something closer to a loss of self.'

One aim of a theory of personal development that emphasises the self-in-relation would be to promote a positive view of women's needs for high-quality relationships, rather than seeing this as a weakness.
Evolutionary psychology This provides a gender-fair theory of personality development and offers perhaps the best understanding of gender-specific trajectories. It suggests that those personality attributes that confer significant benefits in terms of survival and reproduction upon their bearers are transmitted as human-specific, evolved modules of problem-solving relating to these salient life challenges (Cosmides 1992).

Women are physically less powerful than men, so evolution has conferred on them the protection of higher levels of fear. This is encoded genetically through temperamental differences that prevent women engaging in life-threatening physical combat with men (McLean 2009). Girls have a biologically rooted temperamental vulnerability of negative affectivity - a combination of psychological instability, proneness to anxiety, anger and sadness (Rothbart 2006). Lower levels of regulative capacities compared with boys in managing negative affectivity (Muris 2007) lead to higher rates of internalising symptoms in girls (Lonigan 2001).

These symptoms are related to negative perceptions and ruminations, dysphoria and anxiety, and a tendency to internalise negative and destructive impulses. I believe that these may well be the reasons why much of women's psychopathology is experienced and manifested through their bodies: an 'embodiment of emotions'. It is their bodies that act as containers of negative affects and impulses - the so-called 'inner space' (Erikson 1968). Thus, women display higher rates of self-harm, eating disorders, somatisation disorders, conversion and dissociative disorders. These disorders are often chronic, with episodes of acute exacerbations during periods of heightened reactivity to environmental stressors.

Biosocial high-risk periods Two kinds of interaction appear to underlie feminine psychopathology and the timing of onset. Biological predispositions of the nature described above interact with evolutionarily salient life stressors to produce typical manifestations of psychopathology. Such salient periods of high stress occur at four distinct life phases:

1 during early childhood, abuse can lead to a distortion of attachment that installs a disordered template governing future relationships;

2 during adolescence, the onset of female fecundity with menarche signals the need for self-care and attachment related to greater gender socialisation and separation, and often the experience of sexual abuse;

3 in pregnancy, the need for maternal instinctual tasks postpartum provides high biosocial risks; 
4 at the onset of menopause or an analogous condition that removes capacity to bear children (e.g. hysterectomy, amenorrhoea; Welldon 1991).

Thus, the woman's 'biological clock', with associated socially meaningful tasks (even if not always desired by the woman) interacts with 'inner space' such that there are different aetiological factors leading to the onset and exacerbation of psychological disorders during these periods (McLean 2009). Most disorders with high rates of prevalence in women in secure care such as eating disorders, substance misuse, self-harm, offending, sexual perversions and abuse of dependent children (Welldon 1991; Pedersen 2004; McLean 2009) occur during these 'biosocial high-risk' periods.

Treatment journey for women patients in secure care

This section suggests a proposed care pathway for women patients in secure care, which incorporates some of the principles of practice highlighted in this article. During the early stages of treatment the women will require maintenance of a safe environment, containment and 'therapeutic holding'. They may be able, when sufficiently contained and held, to engage in limited therapeutic interventions, such as social problem-solving. During this phase the staff group should have received training in the management of self-harm and the associated risks, and be aware of the potential impact on them of repeated exposure to trauma through witnessing self-harm. It is also important that they have the facility to explore issues of malignant alienation, power and control in reflective staff supervision and support. They will also require training in the specific treatment approaches offered. Subsequently, it may be possible to engage the women in more trauma-informed cognitive-behavioural therapy (CBT) work and/or dialectical behaviour therapy (DBT) work (focused on cognitive skills) and individual body-centred approaches (focused on somatosensory experiential aspects), with support from the clinical team. In addition to specific training, staff will require individual and group supervision and reflective practice to ensure that a consistent approach with sustainable boundaries is provided.

As the women enter a phase of exploration and change, they can complete specific work, such as arson treatment programmes and substance misuse and mental health awareness schemes. Staff will require training in such approaches and should be able to manage possible relapses while such exploratory work is taking place. It may also be possible to facilitate interpersonal exploration and staff should have the requisite skills to provide this, together with the maintenance of previously acquired coping strategies and the beginning of relapse prevention work.

At the end of the treatment journey, women require ongoing support to maintain the new sets of skills that they have acquired, together with some preparation for the next stage of their care pathway. Staff should have the capacity to assist women in transferring these new skills to different environments and to promote the formulation of feasible plans for the future. They should be able to provide relapse prevention work that includes exposure to potential destabilisers and plans for dealing with periods of increased stress. It is imperative that at this stage staff can manage the change inherent in discharge, promote the establishment of future support networks and create a positive transition to the next stage of care for the women.

\section{Future needs for service development}

Gender-specific and relational safety needs

Gender-specific approaches There have been significant changes since the gender-sensitive agenda was established and promoted at the end of the 20th century. However, much still needs to be done before women in secure services nationally receive the same service as men in terms of the range, quality and nature of services delivered within the least restrictive environments. Indeed, the same could be said about generic mental health services for women in England. These factors must underpin any intervention approach that services adopt. It is evident that neither pharmacotherapy nor traditional CBT, DBT or psychoanalysis alone is sufficient to capture the key determinants of what service users and gender-sensitive providers require.

The challenge is to incorporate innovative, creative ways to make the lives of these women meaningful, perhaps by adopting the principles of positive psychology (Seligman 1990) that drive offender intervention strategies such as the 'good lives' model and 'self-regulation' models (Ward 2006). There is a key role that occupational therapy services can play, but only if these interventions are incorporated within an integrated plan of holistic care that caters to the woman, as opposed to her diagnostic labels. The traditional cognitive therapies offered by many women's services are provided within predominantly behavioural programmes regulated by reinforcement and containment with medication and/or structural security approaches such as intensive care and seclusion.

The government's new vision for mental health services - early detection and preventative work, public health psychiatry and mental health 
services, developing resilience and recovery, and providing holistic care to service users - is based on principles that are embedded in everyday practice in gender-sensitive and relationally enhanced secure service pilots.

Relational safety The now-defunct National Service Frameworks taught us some key lessons. The strategy was developed to improve services generally for adults with mental health problems. It identified key areas and developed specialist services (early intervention in psychosis, assertive outreach, home treatment, crisis management, etc.), which provided many good practice standards that are now embedded in generic mental health services. Gender-specific and relationally enhanced services could make a similar contribution in the development of specialist secure services that can inform practice across all layers of mental health services in the UK - in hospitals and in prisons. Service provision must be centred on relational aspects of care and thus attachment-based or attachment-informed approaches are imperative. The critical features that set women's secure services apart were jointly endorsed by those enabling care for women and service users themselves in a national audit (Parry-Crooke 2009; Box 5).

\section{Workforce training and development}

The therapeutic aim of gender-specific and relationally 'enhanced' services is to develop an understanding of the woman patient's complex psychopathology that is shared with the patients and is meaningful to them. Ideally, all psychiatric trainees should receive training in basic gender awareness and sensitivity, highlighting the differences between the genders that have been identified. This could be incorporated as part of a general induction process or during specific rotations. In addition, they need to be well versed in the particular presenting features of women with complex needs, e.g. factors underlying self-harm,

BOX 5 Key characteristics of an ideal women's services

- Relational security: therapeutic relationship with staff

- Trust and positive expectations of self and others

- Empowerment and reduction of social isolation

- Meaningful days and daily staff support

- Holistic approach: person-centred

- Offering a range of interventions to meet emotional needs

(Parry-Crooke 2009) capacity for cruelty towards themselves and dependent others, need for a sense of belonging and safety in emotionally 'containing' relationships. It is imperative that trainees who intend to work in a secure setting acquire an understanding of relational security, whichever gender they intend to provide care for in the longer term. Although this is a particular focus in services for women, it is likely that in the longer term these principles will be extended across all secure services.

However, the establishment of a service focused on relational containment and security has significant implications and challenges for staff at all levels. At the level of recruitment, staff not only should have the requisite clinical skills, but also should have made an active choice to work with women. In addition, they should have a good understanding of gender issues and the nature of the disempowerment that many women will have experienced. Staff should undergo an appraisal process that understands their needs, both in terms of individual training and the benefit to the service.

As part of this process staff must develop a high degree of self-awareness. They have to consider in detail the implications of their own interpersonal styles, life experiences and core beliefs in the context of providing relational security. There must be ample opportunities for reflective practice, which facilitates frank discussions of interpersonal difficulties in a non-judgemental way. Such a focus must encompass regular, systematic, individual supervision for all staff, together with the opportunity for team-based supervision and support. There will be high levels of anxiety expressed in both the patient and staff group. If staff find it difficult to contain their anxieties, confidential stress counselling should be available and be sensitive to the particular issues raised.

It is to be hoped that with greater training and awareness (Box 6) the anxieties of individuals new to secure services for women will be reduced in line with those of staff who are empowered to work in such services through the training and supervision described above. If secure services for women are viewed more positively in the future, trainees will be more likely to make an active choice to commit to working with women patients as a career.

\section{Standardised approaches and evaluative research}

A successful evolution of secure women's services will involve the identification and management of core women-centred factors and seek to standardise relational security as the predominant mode of managing such services. Efforts should be made to eliminate variation in the quality and nature of treatment provided in the NHS and independent 
BOX 6 Recruitment, retention, training and supervision of staff in gender-specific relationally enhanced services

- Staff have chosen to work in gender-specific service and have necessary skills

- Staff are aware of gender-specific issues, themes of attachment, disempowerment and trauma

- There are regular multidisciplinary meetings and forums and good channels of communication

- There should be opportunities for reflective practice and non-judgemental exploration of interpersonal difficulties

- There should be individual and group supervision, staff support and supervision procedures

sectors, and within different geographical regions. There should be greater emphasis on establishing a gender-specific research base, and the routine collection of data by the Department of Health in the pilot services, when analysed, will be of great value in this regard.

Clinicians also have a responsibility to develop clinical research strategies and collect data routinely as part of their service evaluation. This will facilitate bottom-up development of a 'what works and how' literature base, which can inform others. Some strategies in medium secure services can be informed by practices within high secure care, where the majority of those now moving down the security ladder have been for years. However, care must be exercised in implementing approaches that are successful in high security as they may not be the most appropriate strategy in medium security, owing to differences in the emphasis and nature of the security provided, the stage of treatment and rehabilitation of the patients, and the resources available in the medium secure setting. As part of this process, local services need to ensure that managed clinical networks are developed that anticipate the capacities of the differing parts of the networks and can integrate care. This determines the requirements of each part and is particularly important when considering the capacity for working at the interfaces between services, settings and agencies.

Transitions are a period of high risk, and care must be taken to ensure that they are as seamless and well-managed as practicable. Allowing patients ample opportunities to meet with the new team to work through issues of perceived rejection and abandonment by their previous carers and to help them understand the nature and purpose of MCQ answers their self-sabotaging behaviours in a new setting can be of great therapeutic value.

\section{References}

American Psychiatric Association (1994) Diagnostic and Statistical Manual of Mental Disorders (4th edn) (DSM-IV). APA.

American Psychiatric Association (2004) Diagnostic and Statistical Manual of Mental Disorders (4th edn, Text Revision) (DSM-IV-TR). APA.

Bartlett A (2009) Gender, crime and violence. In Forensic Mental Health. Concepts, Systems and Practice (eds A Bartlett, G McGauley): 53-66. Oxford University Press.

Bartlett A, Hassell Y (2001) Do women need special secure services? Advances in Psychiatric Treatment 7: 302-9.

Bland J, Mezey G, Dolan B (1999) Special women, special needs. Descriptive study of female special hospital patients. Journal of Forensic Psychiatry 10: 34-5

Campbell A, Muncer S, Bibel D (2001) Women and crime. An evolutionary approach. Aggression \& Violent Behaviour 6: 481-97.

Chesney-Lind M (1997) The Female Offender: Girls, Women and Crime. Sage Publications.

Coid J, Kahtan N, Gault S, et al (2000) Women admitted to secure forensic psychiatry services. I. Comparison of women and men. Journal of Forensic Psychiatry 11: 275-95.

Cosmides L, Tooby J, Barkow J (1992) Evolutionary psychology and conceptual integration. In The Adapted Mind: Evolutionary Psychology and the Generation of Culture (eds J Barkow, L Cosmides, J Tooby): 3-18. Oxford University Press.

Department of Health (1992) Report of the Committee of Inquiry into Complaints about Ashworth Hospital (Vols 1 \& 2). HMSO.

Department of Health (2000a) Secure Futures for Women: Making a Difference. Department of Health.

Department of Health (2000b) Safety, Privacy and Dignity in Mental Health Units. NHS Good Practice Guide. Department of Health.

Department of Health (2000c) Tilt Review of Security in High Secure Hospitals. Department of Health.

Department of Health (2002) Women's Mental Health: Into the Mainstream. Strategic Development of Mental Health Care for Women. Department of Health.

Department of Health (2003) Mainstreaming Gender and Women's Mental Health. Implementation Guidance. Department of Health.

Department of Health (2009) See, Think, Act: Your Guide to Relational Security. Department of Health.

Erikson E (1968) Identity. Youth and Crisis. Norton.

Farrington DP, Painter KA (2004) Gender Differences in Risk Factors for Offending (Research Findings No. 196). Home Office.

Home Office (2006) Criminal Statistics 2005. Research and Development Statistics.

Home Office (2007) Population in Custody, England and Wales 2006. Research and Development Statistics.

Jurik N, Winn R (1990) Gender and homicide. A comparison of men and women who kill. Violence and Victims 5: 227-42.

Kaplan AG, Surrey JL (1984) The relational self in women. Developmental theory and public policy. In Women and Mental Health Policy (ed LE Walker): 59-94. Sage Publications.

Kruttschnitt C (1993) Violence by and against women. A comparative and cross-national analysis. Violence and Victims 8: 253-70.

Lart R, Payne S, Beumont B, et al (1999) Women and Secure Psychiatric Services. A Literature Review. Report to the NHS Centre for Reviews and Dissemination. University of York NHS Centre for Reviews and Dissemination.

Liem M, Koenraadt F (2008) Filicide: a comparative study of maternal versus paternal child homicide. Criminal Behaviour \& Mental Health 18: $166-76$.

Lonigan C, Phillips B (2001) Temperamental influences on the development of anxiety disorders. In The Developmental Psychopathology of Anxiety (eds M Vasey, R Dadds): 60-91. Oxford University Press. 
Lynam D, Widiger T (2007) Using a general model of personality to understand sex differences in the personality disorders. Journal of Personality Disorders 21: 583-602.

Maden A, Scott F, Burnett R, et al (2004) Offending in psychiatric patients after discharge from medium secure hospitals. Prospective national cohort study. BMJ 328: 1534

Maden A, Skapinakis P, Lewis G, et al (2006) Gender differences in reoffending after discharge from medium-secure units. National cohort study in England and Wales. British Journal of Psychiatry 189: 168-72.

McLean C, Anderson E (2009) Brave men and timid women? A review of the gender differences in fear and anxiety. Clinical Psychology Review, 29: $496-505$

Miller JB (1986) Toward a New Psychology of Women. Penguin.

Milne S, Barron P, Fraser K, et al (1995) Sex differences in patients admitted to a regional secure unit. Medicine, Science and Law 35: $57-60$.

Muris P, Meesters C, Blijlevens P (2007) Self-reported reactive and regulative temperament in early adolescence. Relations to internalizing and externalizing problem behaviour and 'Big Three' personality factors. Journal of Adolescence 30: 1035-49.

Parry-Crooke G, Stafford P (2009) My Life: In Safe Hands? Summary Report of an Evaluation of Women's Medium Secure Services. London Metropolitan University.

Pedersen C (2004) Biological aspects of social bonding and the roots of human violence. Annals of New York Academy of Sciences 1036: 106-27.

Prins H (1982) Criminal Behaviour. An Introduction to Criminology and the Penal System. Tavistock.

Rothbart M, Bates J (2006) Temperament. In Handbook of Child Psychology. Vol. 3. Social, Emotional and Personality Development (eds N Eisenberg, W Damon, RM Lerner): 99-166. Wiley.

Sahota S, Davies S, Duggan C, et al (2009) Women admitted to medium secure care. Their admission characteristics and outcome as compared with men. International Journal of Forensic Mental Health 9: 110-7.
Sarkar J (2011) Public health psychiatry in criminal justice states. In Current Topics in Psychiatry (eds N Agarwal, R Gaind). PalgraveMacmillan (in press).

Seeman M (1995) Conclusion. In Gender \& Psychopathology led M Seeman): 377. American Psychiatric Publishing.

Seligman M (1990) Learned Optimism. Knopf Books.

Smith J, Parker J, Donovan M (1991) Women admissions to a regional secure unit. Journal of Forensic Psychiatry 2: 97-102.

St Andrew's Healthcare (2009) Forensic Directory. St Andrew's Healthcare.

Steels M, Roney G, Larkin E, et al (1998) Discharged from special hospitals under restriction. A comparison of the fates of psychopaths and the mentally ill. Criminal Behaviour \& Mental Health 8: 39-55.

Steffensmeier D (1993) National trends in female arrests 1960-1990. Assessment and recommendations for research. Journal of Quantitative Criminology 9: 411-41.

Tyrer P, Bateman AW (2004) Drug treatment for personality disorders. Advances in Psychiatric Treatment 10: 389-98.

Ward T, Gannon T (2006) Rehabilitation, aetiology and self-regulation. The Good Lives model of sexual offender treatment. Aggression \& Violent Behaviour 11: 77-94.

Warner L, Ford R (1998) Conditions for women in in-patients psychiatric units. The Mental Health Act Commission 1996 national visit. Mental Health Care 1: 225-8.

Weizmann-Henelius G, Viemero V, Eronen M (2003) The violent female perpetrator and her victim. Forensic Science International 133: 197-203.

Welldon EV (1991) Psychology and psychopathology in women. A psychoanalytic perspective. British Journal of Psychiatry 158 (suppl 10): 85-92.

Women In Secure Hospitals (1999) Defining Gender Issues: Redefining Womens' Services. WISH.

Women in Secure Hospitals (2000) Consultation with Women Patients in Secure Mental Health Settings. Report on Independent Evaluation. WISH.

\section{MCOs}

Select the single best option for each question stem

\section{With regard to gender differences in} mental health:

a women are more likely to misuse illicit substances

b schizophrenia has a better prognosis in women

c women have a higher incidence of borderline personality disorder

$\mathrm{d}$ men have lower rates of antisocial and paranoid personality disorder

e women have a lower prevalence of mood disorders.

2 Gender differences in crime:

a men are more likely than women to be convicted of violent offences

b women are more likely to commit offences of serious violence

c women prisoners are less likely to have a diagnosis of personality disorder

d women are more likely to have been employed before imprisonment e women prisoners are less likely to have children in their care.

3 In secure services:

a women are more likely to be transferred from prison

b women are more likely to be admitted because of self-harm or violence towards care-givers

c women are less likely to be detained on civil sections

d men more commonly have index offences of arson

e women are more commonly prosecuted for institutional violence.

4 In secure services:

a gender-sensitive services have been the norm for many years

b women in enhanced medium secure services more commonly have a primary diagnosis of mental illness

c relational security is not a focus for enhanced medium secure services for women $\mathrm{d}$ the greater focus on relational security does not reflect the differing biopsychosocial development of the genders

e women's enhanced medium secure services developed because of contraction in the high secure estate.

5 With regard to future developments:

a meaningful therapeutic relationships are essential in developing relational security

b treatment approaches should be based on behavioural modification and attachment theory

c patients' egregious behaviours are manipulative and do not convey meaning

d staff training, supervision and support is irrelevant in providing higher quality services

e transition and change is a low risk period for women patients. 